\title{
STRENGTH COMPARISON OF DIFFERENT METHODS OF CLOSING THE ANTERIOR ABDOMINAL WALL DEFECT (EXPERIMENTAL STUDY)
}

DOI: 10.36740/WLek202006126

\author{
Oleksandr Yu. loffe, Tetiana V. Tarasiuk, Mykola S. Kryvopustov, Oleksandr P. Stetsenko, Yuri P. Tsiura, \\ Yuri A. Dibrova, Artem V. Vasilyev \\ BOGOMOLETS NATIONAL MEDICAL UNIVERSITY, KYIV, UKRAINE
}

\begin{abstract}
The aim of the study is to compare the mesh implantation and the strength of the mesh fixation to the anterior abdominal wall by modelling the intraperitoneal onlay mesh repair (IPOM) with and without aponeurotic defect closure.

Materials and methods: The experimental animals were randomly divided into 2 groups of 6 rabbits. In experimental group IPOM was modelled without hernia defect closure, in control group - with its suturing. The macroscopic assessment of the severity of adhesions, histological examination of the removed "anterior abdominal wall - mesh" complex and strength of the mesh fixation to the anterior abdominal wall were performed on the 90th day.

Results: In both groups, the displacement or complete separation of the mesh from the parietal peritoneum was not observed. The extent of adhesion formation in the abdominal cavity and strength of the mesh fixation are comparable in both groups $(p>0.05)$. In the experimental group, the mesh was surrounded by scar tissue, mesothelioma lining was not traced. There were also moderate signs of inflammation, which were not seen in the control group.

Conclusions: The strength of the mesh fixation to the parietal peritoneum and its implantation into the anterior abdominal wall is comparable with or without aponeurotic defect closure during the experimental modelling of IPOM.
\end{abstract}

KEY WORDS: ventral hernia, mesh fixation, defect closure, IPOM

Wiad Lek. 2020;73(6):1217-1222

\section{INTRODUCTION}

Ventral hernias are one of the most common pathologies in surgery. The incidence ranges widely from 2 to $69 \%$ and depends on the type of ventral hernia. A postoperative hernia is observed in $10-20 \%$ of patients undergoing laparotomy. [1-5]

The mesh sutured repair of the hernia defect is a standard procedure for the surgical treatment of ventral hernias during routine surgery. [6] In hernioplasty, the mesh-based repair method has repeatedly demonstrated its effectiveness compared to the suture-based closure techniques in various modifications. The International Endohernia Society notes in its recommendations that the use of meshes in hernioplasty reduces a hernia recurrence rate significantly compared to the defect closure without mesh. [7] The laparoscopic intraperitoneal onlay mesh repair demonstrates a lower percentage of local postoperative complications compared with the open ventral hernia repair. [8] However, laparoscopic surgery does not always involve hernia defect closure. Suwa K et al. emphasises on lack of the research publications that analyse the outcomes of the IPOM procedure with and without hernia defect closure before mesh placement. Hernia defect closure before the IPOM procedure is associated with more favourable surgical outcomes. [9] However, in a study of 134 patients, Gonzalez A et al. observed a higher recurrence rate of $7.5 \%$ in the group without hernia defect closure compared to $1.5 \%$ in the group with hernia defect closure before the IPOM procedure. [10] The availability of mostly comparative studies and a limited sample of patients cannot provide reliable evidence for determining the effectiveness of the IPOM procedure without hernia defect closure. Since the hernia recurrence occurs at the site of separation of the mesh from the anterior abdominal wall, in our experimental study, we investigated the strength of the mesh fixation to the parietal peritoneum by evaluating different closure techniques for hernia repair.

\section{THE AIM}

The aim of the present research is to compare the mesh implantation and the strength of the mesh fixation to the anterior abdominal wall by modelling the IPOM procedure with and without aponeurotic defect closure (a modelled hernia defect).

\section{MATERIALS AND METHODS}

The experimental study evaluates the outcomes of the IPOM technique. The method was modelled and applied to 12 rabbits. The experimental animals (rabbits) were selected based on the possibility of modelling the intra- 
peritoneal placement of a $4 \mathrm{x} 4 \mathrm{~cm}$ composite mesh, which is usually technically difficult in small rodents. All animals were quarantined and kept under standard conditions. The study used rabbits of both sexes, of the Russian Chinchilla breed, with weight from 2.1 to $2.9 \mathrm{~kg}$.

The experimental animals were randomly divided into 2 groups of 6 rabbits. In both groups, surgery was started by performing a laparotomy through a longitudinal incision of the skin along the median line up to $4 \mathrm{~cm}$ long. Fig. 1 The hernia defect, measuring $1 \times 1 \mathrm{~cm}$ along the median line, was modelled in all rabbits in both groups. In the experimental group, the intraabdominal implantation of a 4 $\mathrm{x} 4 \mathrm{~cm}$ composite mesh without hernia defect closure was performed. In both groups, a lightweight polypropylene mesh encapsulated in soluble poly-p-dioxanone (PDS II) was used. On one side, the mesh is covered with oxidized regenerated cellulose, which is placed inside to contact the visceral peritoneum. In this case, there was obtained an overlap of the mesh at least $1 \mathrm{~cm}$ beyond the hernia defect in all directions. In the control group, the intraabdominal hernioplasty was modelled in 6 rabbits and a $4 \mathrm{x} 4 \mathrm{~cm}$ composite mesh was implanted together with hernia defect closure. In both groups, the mesh was fixed with separate interrupted sutures at 8 sites transaponeurotically to the parietal peritoneum. Fig. 2 The restoration of the integrity of the anterior abdominal wall was carried out in layers by applying a continuous suture to the aponeurosis of the rectus abdominis and to the skin. At the end of surgery, the wound was dressed with an aseptic bandage and a protective band was applied.

All surgical interventions were carried out in compliance with the requirements of the European Convention for the Protection of Vertebrate Animals used for Experimental and other Scientific Purposes (1986) and Scientific and Practical Recommendations for the Maintenance of Laboratory Animals and their Operation by the State Pharmacological Centre of the Ministry of Health of Ukraine (2002), which was confirmed by the decision of the Ethics Committee of the Bogomolets National Medical University.

In both groups, the operations were performed under general anaesthesia, with the requirements of aseptic and antiseptic. As premedication, all animals were administered $0.4 \mathrm{ml}$ of a $2 \%$ solution of xylazine hydrochloride. For the purpose of sedation and analgesia, $0.8 \mathrm{ml}$ of a $0.1 \%$ solution of medetomidine hydrochloride was administered. The animals were placed on the operating table with their backs down, with each leg fixed separately. The anterior abdominal wall hair was removed by the method of dry shaving.

At the end of surgery in both groups, all the rabbits were administered $0.8 \mathrm{ml}$ of $0.5 \%$ solution of atipamezole hydrochloride to eliminate sedation. Additionally, all animals were administered ketoprofen $3 \mathrm{mg} / \mathrm{kg}$ subcutaneously for analgesia. Antibiotic prophylaxis was performed by intramuscular injection of cefazolin $1.0 \mathrm{~g} 30 \mathrm{~min}$ before surgery and 12 hours after surgery. All the animals were in a vivarium with a standard mode of stay and food.

In both groups, the animals were removed from the experiment on the 90th day by overdose of drugs for anaes- thesia - intravenous administration of $1.0 \mathrm{~g}$ of sodium thiopental, diluted in $5 \mathrm{ml}$ of $0.9 \%$ sodium chloride solution.

After opening of the abdominal cavity, macroscopic assessment of the severity of adhesions was performed. For this purpose, we used the classification, proposed by Zühlke et al. in 1990, to evaluate the severity of adhesions in the abdominal cavity in experimental animals. [11] The severity of adhesions is scored according to the macroscopic evaluation and classified as:

Grade I - fibrin deposits, tiny filmy adhesions or sporadic adhesions between the organs that can be separated by blunt dissection;

Grade II - filmy adhesions that can be can be separated by blunt dissection, but there are also some adhesions with initial vascularization, which can only be separated by sharp dissection;

Grade III - adhesions with clear vascularization, dense and filmy adhesions that can only be separated by sharp dissection;

Grade IV - dense and severe adhesions between the organs that can only be separated by sharp dissection; damage of organs is hardly preventable during surgical separation.

The presence of displacements and partial or complete separation of the mesh from the parietal peritoneum were also evaluated.

After separation of adhesions by partially blunt and partially sharp dissection, the material was collected for the histological examination and evaluation of the strength of the mesh implantation into the anterior abdominal wall layer. The "anterior abdominal wall - mesh" complex was removed completely together with the adjacent tissues of the muscular-aponeurotic layer of the anterior abdominal wall. The fixation of the material was carried out with $10 \%$ formalin solution in water.

The microscopic evaluation of mesh implantation into the tissues of the anterior abdominal wall was performed by histological examination of the removed "anterior abdominal wall - mesh" complex.

The strength of the mesh fixation to the anterior abdominal wall of the rabbit was evaluated by the maximum shear stresses at the time of destruction of the sample for the "anterior abdominal wall - mesh" complex. The shear stress was taken as $\mathrm{t}^{*}=\frac{P}{A}$, where $\mathrm{P}$ is the applied force, $\mathrm{A}$ is the contact area of the mesh and the muscle tissue of the anterior abdominal wall, $A=a \cdot b$. Fig. 3

$\mathrm{a}$ - the length of the fusion between mesh and the fragment of the anterior abdominal wall, $b$ - the width of the fusion between mesh and the fragment of the anterior abdominal wall, $\mathrm{c}$ - the thickness of mesh, $\mathrm{d}$ - the length of the free edge of the fragment of the anterior abdominal wall, $\mathrm{e}$ - the thickness of the fragment of the anterior abdominal wall (muscular tissue), $\mathrm{f}$ - the length of the free edge of mesh, $\mathrm{P}$ - the direction of the effort applied.

The mechanical strength tests were performed on an PM-05 machine with an electromechanical drive.

\section{RESULTS}

In both groups, no signs of intra- and postoperative complications were observed. 


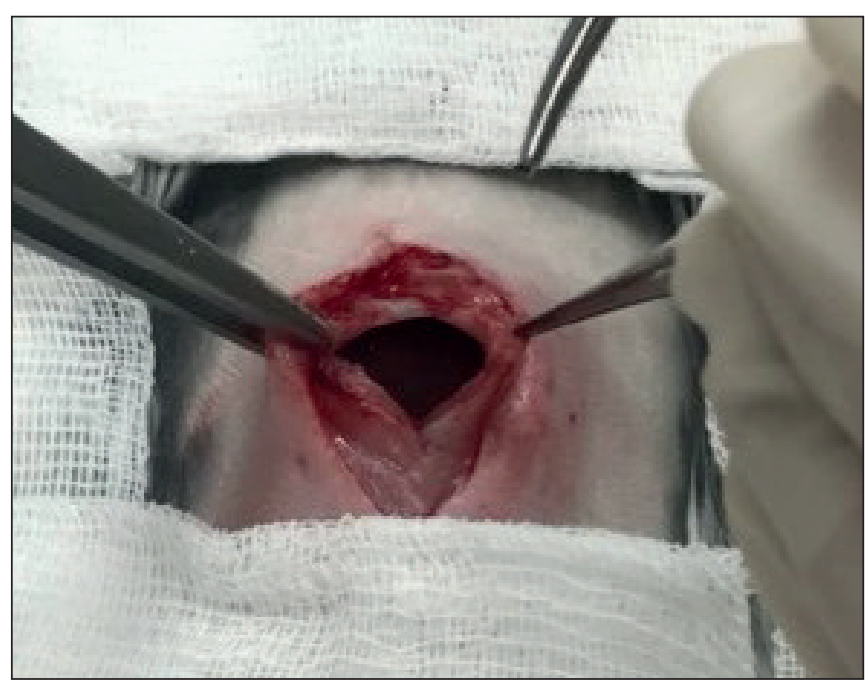

Fig. 1. Intraoperative photo of laparotomy access.

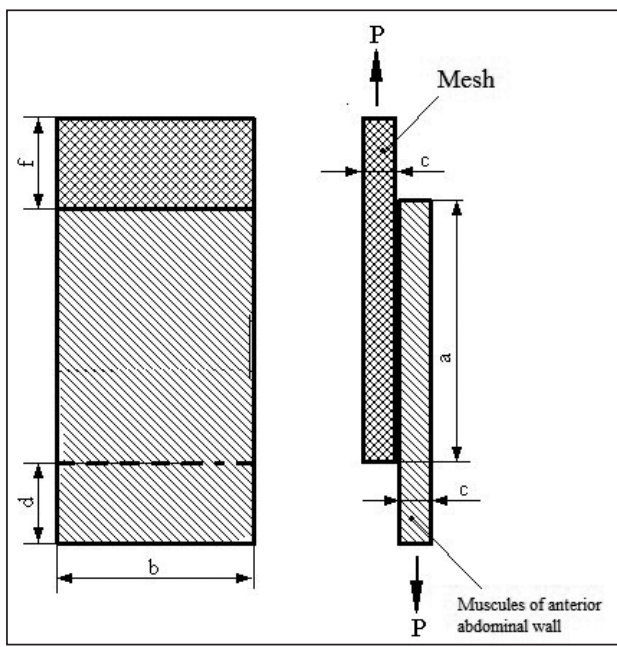

Fig. 3. Scheme of the study of the sample shear.

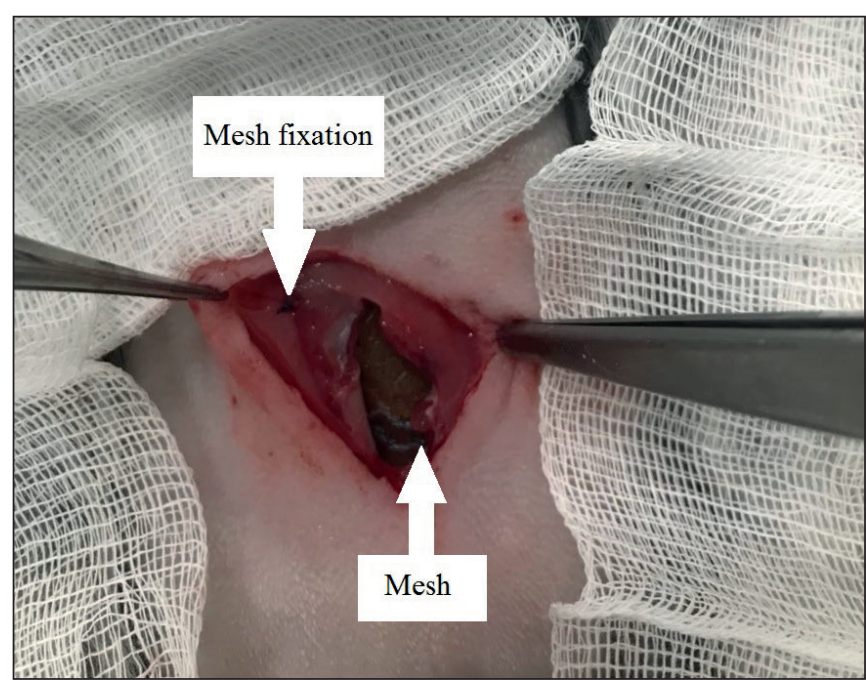

Fig. 2. Intraoperative photo of the mesh fixation to the anterior abdominal wall.

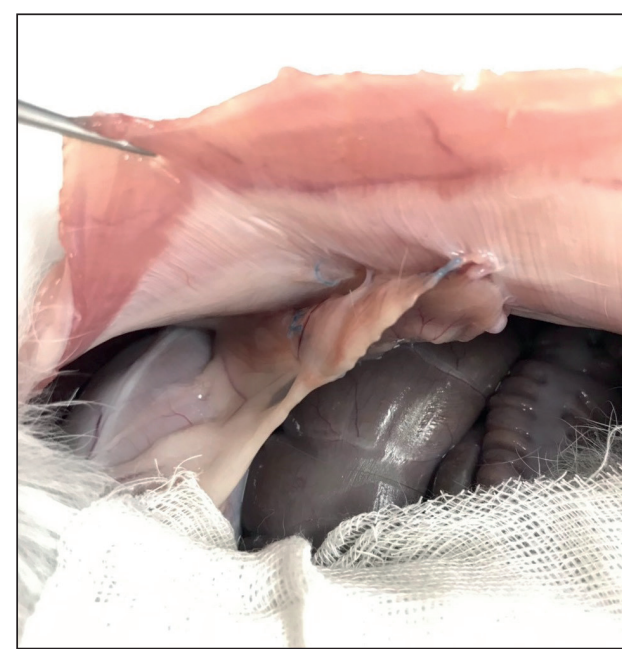

Fig. 4. Photo of partial separation of the mesh from the anterior abdominal wall.

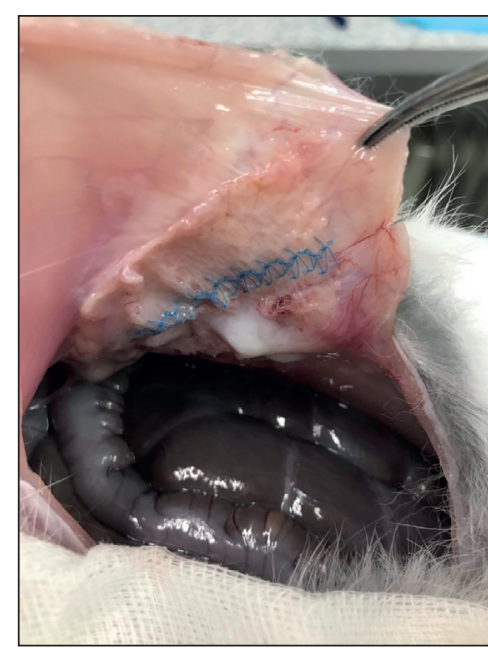

Fig. 5. Photo of the mesh implantation into the anterior abdominal wall layer.

Table I. Characteristics of the severity of adhesions in animals of the experimental and control groups according to the classification of Zühlke et al.

\begin{tabular}{|c|c|c|c|c|c|}
\hline Group & № of rabbit & Grade I & Grade II & Grade III & Grade IV \\
\hline \multirow{6}{*}{$\begin{array}{l}\text { Experimental } \\
\qquad(n=6)\end{array}$} & $1 \mathrm{e}$ & & + & & \\
\hline & $2 e$ & & + & & \\
\hline & $3 e$ & + & & & \\
\hline & $4 \mathrm{e}$ & + & & & \\
\hline & $5 e$ & + & & & \\
\hline & $6 e$ & & + & & \\
\hline \multirow{6}{*}{$\begin{array}{c}\text { Control } \\
(n=6)\end{array}$} & $1 c$ & + & & & \\
\hline & $2 c$ & + & & & \\
\hline & $3 c$ & & & + & \\
\hline & $4 c$ & & + & & \\
\hline & $5 c$ & & + & & \\
\hline & $6 c$ & + & & & \\
\hline
\end{tabular}

On the 90th day in both groups, any displacement or complete separation of the mesh from the parietal peritoneum was not seen. In the control group in 1 case, there was an area of partial separation of the mesh from the parietal peritoneum. Fig. 4 This might be due to the limited number of stitches that were put on and the lack of the possibility of fixation by the double crown method in the experiment. In all animals of the experimental group and in 5 animals 


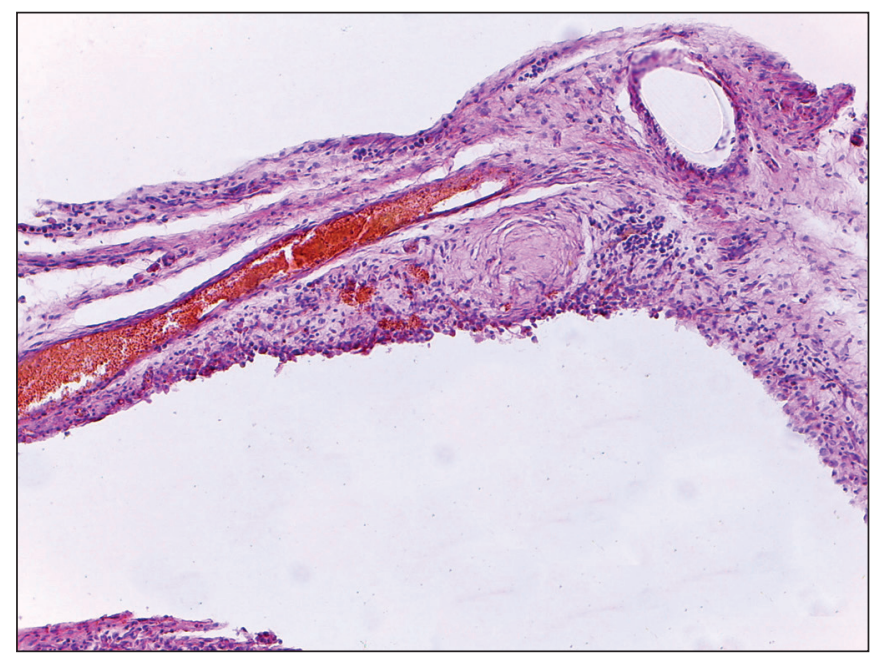

Fig. 6. Microphoto of tissue section around the mesh in the experimental group. Observation period 90 days. Staining with hematoxylin and eosin. Magnification 100.

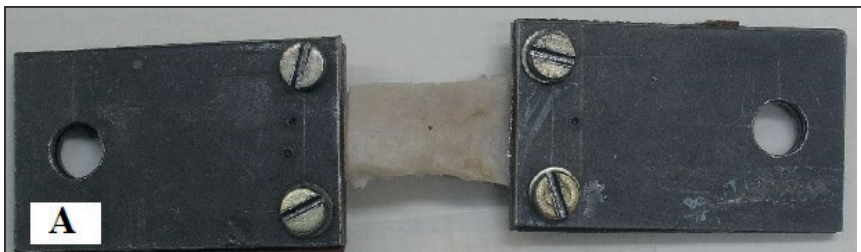

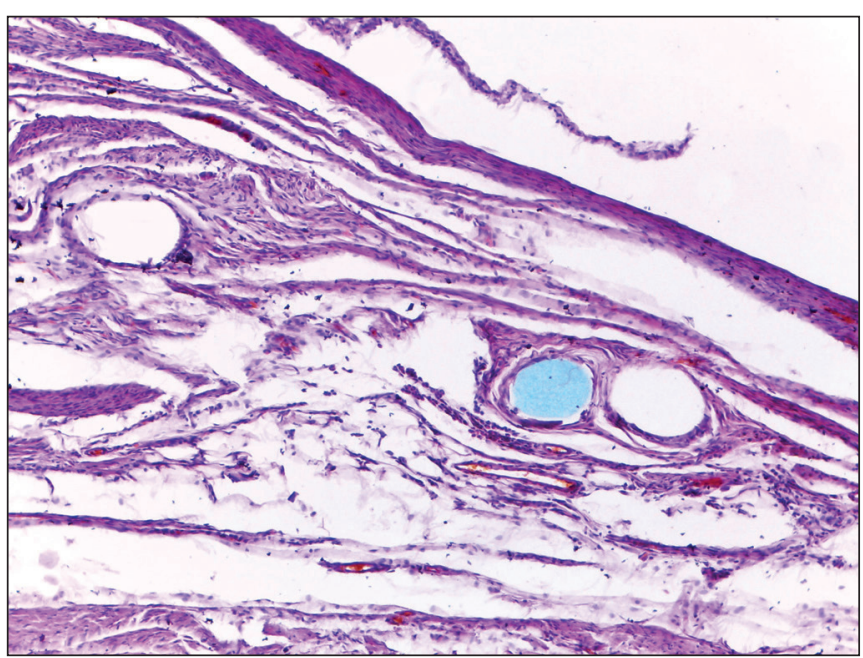

Fig. 7. Microphoto of tissue section around the mesh in the control group. Observation period 90 days. Staining with hematoxylin and eosin. Magnification 100.

Fig. 8. The "anterior abdominal wall - mesh" complex before $(A)$ and after (B) destruction.

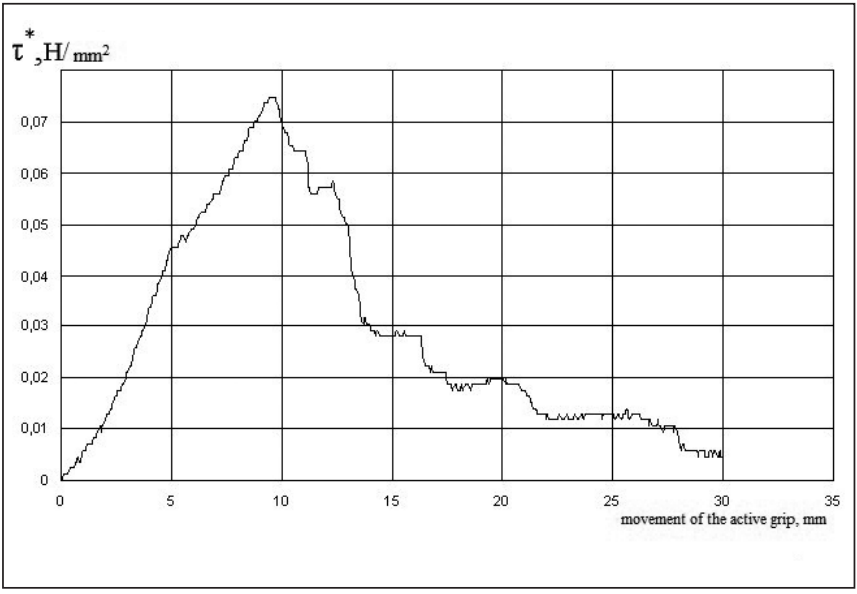

Fig. 9. Schedule of stretching and destruction of the "anterior abdominal wall - mesh" sample complex in the experimental group.

of the control group, macroscopically complete fusion of the implant with the parietal peritoneum of the anterior abdominal wall was observed. Fig. 5

Table I presents the comparative characteristics of the severity of adhesions in the abdominal cavity according to the macroscopic evaluation in the experimental and control groups, based on the classification of Zühlke et al. (1990).

In the experimental group, 3 rabbits (50\%) had fibrin deposits on the visceral surface of the mesh, 3 rabbits (50\%) had filmy adhesions with loops of the small intestine and the large cap, which were easily destroyed by the instrument. In the control group, in $1(16.6 \%)$ case, dense

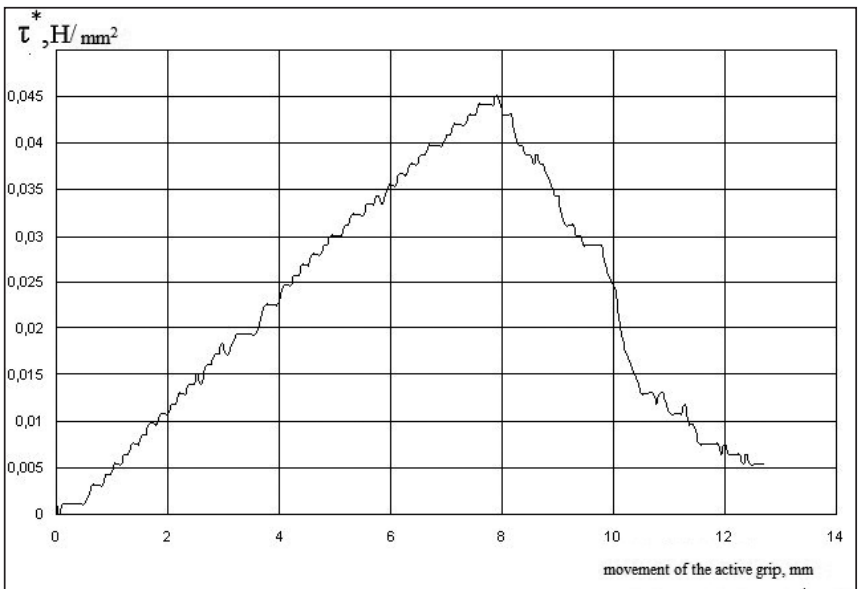

Fig. 10. Schedule of stretching and destruction of the "anterior abdominal wall - mesh" sample complex in the control group.

adhesions were observed between the mesh and the strand of the large cap - grade III. Fig. 4 However, in our opinion, this can be explained by partial separation of the mesh from the parietal peritoneum, observed in this particular case, and prolonged traumatization of the free edge of the mesh of the visceral peritoneum, since adhesions were formed at the site of the free edge of the mesh and were not seen in the place of its complete fusion with the parietal peritoneum. Filmy adhesions were detected in 2 (33.4\%) cases, and in $3(50 \%)$ cases there were seen the layers of fibrin sporadically distributed on the surface of the mesh implanted into the anterior abdominal wall. The results 
of macroscopic evaluation allow us to conclude that the extent of adhesion formation in the abdominal cavity is comparable in both groups ( $\mathrm{p}>0.05)$.

The histological examination of the samples of the "anterior abdominal wall-mesh" complex showed that the experimental group formed a significant amount of scar tissue at the site of the mesh. The implantation area was infiltrated with lymphocytes, especially around the mesh. Mesothelioma lining was not traceable. At the implantation site, there were observed a few dilated blood vessels accommodating increased blood volume. In some areas there were accumulations of macrophages with increased volume of cytoplasm. Moderate signs of inflammation were noted at the site of the mesh. Fig. 6

In the control group, the following changes were observed: mesothelioma lining on the side of the abdominal cavity was absent in some areas where adhesions were seen. Each filament of the mesh was surrounded by a connective tissue capsule. The capsules were mostly thin, but the formation of thick inhomogeneous capsules was noted around some filaments. The mesh sprouted with a mature dense connective tissue. The connective tissue was predominantly layered at the site of the mesh, and moderately infiltrated by lymphocytes and neutrophilic granulocytes near the filaments. Some capsules contained small, mostly flattened, giant foreign body cells or single activated macrophages. No signs of abnormal changes were found in the surrounding tissues. Fig. 7

In accordance with the obtained findings of histological examinations, it was found out that, in the experimental group, the mesh was surrounded by a connective tissue and it was fixed to the anterior abdominal wall. However, mesothelioma lining was not traced, and the mesh was surrounded by scar tissue. In addition, in the experimental group there were observed moderate signs of inflammation, which were not seen in the control group. The observed changes could result from the contact between the edges of a truncated unclosed aponeurosis and the parietal surface of the mesh during a hernia defect modelling.

The assessment of the strength of the mesh fixation to the anterior abdominal wall showed that the average value of maximum shear stress $\left(\mathrm{t}_{\max }^{*}\right)$ for the "anterior abdominal wall - mesh" complex was $0.059 \pm 0.02 \mathrm{MPa}$ in the experimental group and $0.066 \pm 0.03 \mathrm{MPa}$ in the control group. According to the results of the study, the strength of mesh fixation was significantly comparable in both groups $(\mathrm{p}>$ 0.05). Fig. 8, 9, 10

\section{DISCUSSION}

The experiment demonstrated the comparability of the strength of the mesh fixation to the anterior abdominal wall with and without aponeurotic defect closure during modelling of the IPOM procedure. It is not possible to extrapolate the results of the mesh fixation experiment to patients with hernia with $100 \%$ confidence. The standard IPOM technique involves fixing the mesh by the double crown method and the presence of a hernia sack, that could not be reproduced in the exper- iment. These differences can affect the implantation of the mesh. Zeichen MS et al. indicated that the recurrence rate in patients without hernia defect closure was $19.18 \%$, while with hernia defect closure it was 6.25\%. [12] However, he noted that the difference did not reach statistical significance. This may be due to the different number of patients sampled to compare the two methods. In an overview study of the effectiveness of IPOM with hernia defect closure, Suwa K et al. indicated the inability to objectively evaluate the difference in effective defect closure and the benefits of its suture-based repair. [9] The results of the existing studies differ in the techniques for the IPOM procedure with hernia defect closure, the type of the mesh selected, the conditions for the study. Therefore, it is currently not possible to reliably determine the benefits of the IPOM procedure that is performed with or without hernia defect closure. Lambrecht JR et al. compared the outcomes of the hernia repair with and without hernia defect closure. The study included 194 patients with primary and postoperative ventral hernias, 107 of whom were enrolled in a randomized controlled multicentre trial, 87 were retrospectively studied. [13] The study did not establish the benefits of long-term outcomes after hernia defect closure using absorbable suture but revealed a higher overall complication rate. The use of 4 different methods for aponeurotic defect closure and mesh fixation (suture-raphe, suture-nonraphe, double crown-raphe, double crown-non-raphe) in 107 patients and the creation of 8 clusters in 4 unbalanced randomization groups did not reveal the benefits of one of the presented surgical methods. There is a need for randomized, controlled, multicentre trials, in which a standardized IPOM technique with and without hernia defect closure is applied to evaluate the effectiveness of each method in a larger sample of patients.

\section{CONCLUSIONS}

The strength of the mesh fixation to the parietal peritoneum and its implantation into the anterior abdominal wall is comparable with or without aponeurotic defect closure during the experimental modelling of intraperitoneal onlay mesh repair.

\section{REFERENCES}

1. Dabbas N., Adams K., Pearson K. et al. Frequency of abdominal wall hernias: is classical teaching out of date? JRSM Short Rep. 2011;2(1):1-6. doi:10.1258/shorts.2010.010071.

2. Seiler C., Bruckner T., Diener M. et al. Interrupted or Continuous Slowly Absorbable Sutures For Closure of Primary Elective Midline Abdominal Incisions. Ann Surg. 2009;249(4):576-582. doi:10.1097/ sla.0b013e31819ec6c8.

3. Mudge M., Hughes L.. Incisional hernia: A 10 year prospective study of incidence and attitudes. British Journal of Surgery. 1985;72(1):70-71. doi:10.1002/bjs.1800720127.

4. Walming S., Angenete E., Block M. et al. Retrospective review of risk factors for surgical wound dehiscence and incisional hernia. BMC Surg. 2017;17(1). doi:10.1186/s12893-017-0207-0.

5. Alnassar S., Bawahab M., Abdoh A. et al. Incisional hernia postrepair of abdominal aortic occlusive and aneurysmal disease: five-year incidence. Vascular. 2012;20(5):273-277. doi:10.1258/vasc.2011.0a0332. 
6. Rutkow I. Demographic and socioeconomic aspects of hernia repair in the United States in 2003. Surgical Clinics of North America. 2003;83(5):1045-1051. doi:10.1016/s0039-6109(03)00132-4.

7. Bittner R., Bingener-Casey J., Dietz U. et al. Guidelines for laparoscopic treatment of ventral and incisional abdominal wall hernias (International Endohernia Society (IEHS) - Part 1. Surg Endosc. 2013;28(1):2-29. doi:10.1007/s00464-013-3170-6.

8. Sauerland S., Walgenbach M., Habermalz B. et al. Laparoscopic versus open surgical techniques for ventral or incisional hernia repair. Cochrane Database of Systematic Reviews. 2011. doi:10.1002/14651858. cd007781.pub2.

9. Suwa K., Okamoto T., Yanaga K. Closure versus non-closure of fascial defects in laparoscopic ventral and incisional hernia repairs: a review of the literature. Surg Today. 2015;46(7):764-773. doi:10.1007/s00595015-1219-y.

10. Gonzalez A., Romero R., Seetharamaiah R. et al. Laparoscopic ventral hernia repair with primary closure versus no primary closure of the defect: potential benefits of the robotic technology. The International Journal of Medical Robotics and Computer Assisted Surgery. 2014;11(2):120-125. doi:10.1002/rcs.1605.

11. Zühlke H.V., Lorenz E.M., Straub E.M. et al. Pathophysiology and classification of adhesions. Langenbecks Arch Chir Verh Dtsch Ges Chir. 1990, Suppl 2: 1009-1016.

12. Zeichen M., Lujan H., Mata W. et al. Closure versus non-closure of hernia defect during laparoscopic ventral hernia repair with mesh. Hernia. 2013;17(5):589-596. doi:10.1007/s10029-013-1115-6.

13. Lambrecht J., Vaktskjold A., Trondsen E. et al. Laparoscopic ventral hernia repair: outcomes in primary versus incisional hernias: no effect of defect closure. Hernia. 2015;19(3):479-486. doi:10.1007/s10029-015-1345-x.
The work is a fragment of the planned research work of the Department of General Surgery No2 of Bogomolets National Medical University "Implementation of minimally invasive surgical techniques in the treatment of pathologies of the abdominal cavity, anterior abdominal wall, morbid obesity by fast track technique" (State registration number 0118U000147).

\section{ORCID and contributorship:}

Oleksandr Yu. Ioffe - 0000-0002-1306-7920 A,F

Tatiana V. Tarasiuk, ORCID: 0000-0001-6629-3908 A,B,D,E,F

Mykola S. Kryvopustov, ORCID: 0000-0003-4978-4873 C,D

Oleksandr P. Stetsenko ORCID: 0000-0002-2219-653X ${ }^{B}$

Yuri P. Tsiura ORCID: 0000-0001-6651-8564 ${ }^{B}$

Yuri A. Dibrova, ORCID: 0000-0002-2833-1667 ${ }^{E}$

Artem V. Vasilyev, ORCID: 0000-0003-1367-9780 ${ }^{E}$

\section{Conflict of interest:}

The Authors declare no conflict of interest.

\section{CORRESPONDING AUTHOR}

\section{Tetiana V. Tarasiuk}

Department of General Surgery No2,

Bogomolets National Medical University

Taras Shevchenko Boulevard, 13, 01601, Kyiv, Ukraine

tel: +380677126840

e-mail: tara-tanya@ukr.net

Received: 02.03 .2020

Accepted: 05.05 .2020 